\title{
SKA synergy with Microwave Background studies
}

Carlo Burigana, ${ }^{a b}$ Paul Alexander, ${ }^{c}$ Carlo Baccigalupi, ${ }^{d}$ Domingos Barbosa, ${ }^{e}$ Alain Blanchard ${ }^{f}$ Adriano De Rosa ${ }^{a}$ Gianfranco de Zotti, ${ }^{g d}$ Fabio Finelli, ${ }^{a}$ Alessandro Gruppuso, ${ }^{a}$ Michael Jones, ${ }^{h}$ Sabino Matarrese,${ }^{i}$ Alessandro Melchiorri, ${ }^{l}$ Diego Molinari, ${ }^{j}$ Mattia Negrello, ${ }^{g}$ Daniela Paoletti, ${ }^{a}$ Francesca Perrotta, ${ }^{d}$ Roberto Scaramella, ${ }^{k}$ and Tiziana Trombetti ${ }^{a}$

a INAF-IASF Bologna, Via Piero Gobetti 101, I-40129, Bologna, Italy

${ }^{b}$ Dipartimento di Fisica e Scienze della Terra, Università degli Studi di Ferrara, Via Giuseppe Saragat 1, I-44100 Ferrara, Italy

${ }^{c}$ Cavendish Laboratory, Cambridge, UK

${ }^{d}$ SISSA, Astrophysics Sector, Via Bonomea 265, 34136 Trieste, Italy

${ }^{e}$ Radioastronomy Group, Instituto de Telecomunicações, Campus Universitario de Aveiro, 3810-183 Aveiro, Portugal

${ }^{f}$ Université de Toulouse, UPS-OMP, IRAP, Toulouse, France

${ }^{g}$ INAF - Osservatorio Astronomico di Padova, Vicolo dellÕOsservatorio 5, Padova, Italy

${ }^{h}$ Department of Physics, Oxford University, Oxford, UK

${ }^{i}$ Dipartimento di Fisica e Astronomia G. Galilei, Università degli Studi di Padova, Via Marzolo 8, 35131 Padova, Italy

${ }^{l}$ Dipartimento di Fisica, Università La Sapienza, P. le A. Moro 2, Roma, Italy

${ }^{j}$ Instituto de Física de Cantabria (CSIC-Universidad de Cantabria), Avda. los Castros s/n, Santander, Spain

${ }^{k}$ INAF - Osservatorio Astronomico di Roma, Via Frascati 33, 00040 Monteporzio Catone (Roma), Italy

E-mail: burigana@iasfbo.inaf.it

The extremely high sensitivity and resolution of the Square Kilometre Array (SKA) will be useful for addressing a wide set of themes relevant for cosmology, in synergy with current and future cosmic microwave background (CMB) projects. Many of these themes also have a link with future optical-IR and X-ray observations. We discuss the scientific perspectives for these goals, the instrumental requirements and the observational and data analysis approaches, and identify several topics that are important for cosmology and astrophysics at different cosmic epochs.

Advancing Astrophysics with the Square Kilometre Array

June 8-13, 2014

Giardini Naxos, Italy 


\section{Introduction}

Although it is not specifically designed for cosmic microwave background (CMB) observations, because of its high resolution and limited high-frequency coverage, the extremely high sensitivity of the SKA (Dewdney et al. 2013, Braun 2014) may be used to address a wide set of themes relevant for cosmology, in synergy with current and future CMB projects. Many of these also have a strong link with the future Euclid ${ }^{1}$ mission and Athena ${ }^{2}$ observations (Takahashi et al. 2015).

In this chapter we will discuss several important themes: (i) the contribution to future highprecision $\mathrm{CMB}$ absolute temperature experiments aimed at detecting spectral distortion, including long wavelength free-free emission linked to cosmological reionization (Sect. 2); (ii) crosscorrelation between CMB and SKA surveys for the analysis of Integrated Sachs-Wolfe (ISW) effect and constraining on dark energy (Sect. 3); (iii) constraining non-Gaussianity with joint analyses of CMB and radiosources (Sect. 3); (iv) primordial magnetic fields (Sect. 4); (v) Galactic foreground studies, linked to component separation in future CMB experiments (Sect. 5).

From the observational point of view, these topics can be divided into two broad categories (Burigana et al. 2004): those relying on precise point source observations and those that require sensitivity to extended structures. The former take advantage of the high resolution and point-source sensitivity naturally offered by interferometry, while the latter require a suitable array design and observational approach, such as a compact configuration implementing short baselines and a high $u v$-space filling factor, mosaicing techniques, and for the largest areas, methods such as the on-thefly-mapping. These approaches, required by a wide set of SKA scientific projects, are still under definition and optimization. ${ }^{3}$ We will identify the corresponding instrumental and observational requirements and data analysis approaches needed to carry out the proposed studies.

\section{SKA contribution to future CMB spectrum experiments}

The current limits on CMB spectral distortions and the constraints on energy dissipation processes in the plasma (Salvaterra \& Burigana 2002) $\left(\left|\Delta \varepsilon / \varepsilon_{i}\right| \lesssim 10^{-4}\right.$ ) are mainly set by the COsmic Background Explorer ${ }^{4}$ (COBE) / Far InfraRed Absolute Spectrophotometer (FIRAS) experiment (Mather et al. 1990, Fixsen et al. 1996). High accuracy CMB spectrum experiments from space, such as the Diffuse Microwave Emission Survey (DIMES) (Kogut 1996, Kogut 2003) at $\lambda \gtrsim 1 \mathrm{~cm}$ and FIRAS II (Fixsen $\&$ Mather 2002) at $\lambda \lesssim 1 \mathrm{~cm}$, have been proposed to constrain (or probably detect) energy exchanges 10-100 times smaller than the FIRAS upper limits. Experiments such as DIMES may probe dissipation processes at early times $\left(z \gtrsim 10^{5}\right)$ resulting in Bose-Einstein-like distortions (Sunyaev \& Zeldovich 1970, Danese \& de Zotti 1980, Burigana et al. 1991) and freefree distortions (Bartlett $\&$ Stebbins 1991) possibly generated by heating mechanisms at late epochs $\left(z \lesssim 10^{4}\right.$ ), before or after the recombination era (Burigana \& Salvaterra 2003) (or possibly cooling processes, see Stebbins \& Silk 1986, although these are disfavoured by Wilkinson Microwave Anisotropy Probe ${ }^{5}$ (WMAP) data). These possibilities have been recently reconsidered in the context of new CMB space missions such as the Primordial Inflation Explorer (PIXIE) (Kogut et al.

\footnotetext{
${ }^{1}$ www.rssd.esa.int/euclid

${ }^{2} \mathrm{http} / / /$ www.the-athena-x-ray-observatory.eu/

${ }^{3}$ See http://www-astro.physics.ox.ac.uk/ hrk/SKA_EXPOSURE.html for a numerical tool.

${ }^{4} \mathrm{http}: / /$ lambda.gsfc.nasa.gov/product/cobe/

${ }^{5} \mathrm{http} / / /$ lambda.gsfc.nasa.gov/product/map/current/
} 
2011) proposed to NASA, which combines high-accuracy polarization and spectrum measurements at $\sim$ degree resolution, and in the possible inclusion of spectral measurements in highly sensitivite polarization $\mathrm{CMB}$ space missions with arcmin resolution, such as the Cosmic Origins Explorer ${ }^{6}$ (COrE) (COrE Collaboration 2011) proposed to the ESA and its possible successors, such as the Polarized Radiation Imaging and Spectroscopy Mission ${ }^{7}$ (PRISM) (PRISM Collaboration 2014).

Calculations of various types of typical distorted spectra (see left panel of Fig. 1) have been presented in various works (e.g. Danese \& Burigana 1994, Procopio \& Burigana 2009, Khatri \& Sunyaev 2012). Improving CMB absolute temperature measures will give a corresponding strengthening of the constraints on physical parameters of various classes of processes (see e.g. PRISM Collaboration 2014 and references therein). Decaying and annihilating particles during the pre-recombination epoch may affect the CMB spectrum, with the exact distorted shape depending on the process timescale and, in some cases, being different from that produced by energy release. This is especially interesting for decaying particles with lifetimes $t_{X} \approx$ few $\times 10^{8}-10^{11} \mathrm{sec}$ (Danese $\&$ Burigana 1994, Chluba \& Sunyaev 2012). Superconducting cosmic strings would also produce copious electromagnetic radiation, creating CMB spectral distortion shapes (Ostriker \& Thompson 1987) that would be distinguishable with high accuracy measurements. Evaporating primordial black-holes provide another possible source of energy injection, with the shape of the resulting distortion depending on the black-hole mass function (Carr et al. 2010). CMB spectral distortion measurements could also be used to constrain the spin of non-evaporating black-holes (Pani \& Loeb 2013). The CMB spectrum could also set constraints on the power spectrum of small-scale magnetic fields (Jedamzik et al. 2000), the decay of vacuum energy density (Bartlett \& Silk 1990), axions (Ejlli \& Dolgov 2014), and other new physics processes. Deciphering these signals is a challenge, but holds the potential for important new discoveries and constraining unexplored processes that cannot be probed by other means.

In addition to the processes discussed above, a certain level of departure of the CMB spectrum from a perfect blackbody is theoretically predicted due to some unavoidable fundamental processes. Cosmological reionization produces electron heating which causes a Comptonization distortion proportional to the fractional amount of energy exchanged during the interaction, characterized by the Comptonization parameter $y(t)=\int_{t_{i}}^{t}\left[\left(\phi-\phi_{i}\right) / \phi\right]\left(k_{B} T_{e} / m_{e} c^{2}\right) n_{e} \sigma_{T} c d t \simeq(1 / 4) \Delta \varepsilon / \varepsilon_{i}$ (where the last equality holds in the limit of small energy injections and integrating over the relevant epochs). Here $\phi(z)=T_{e}(z) / T_{C M B}(z)$, and $\phi_{i}=\phi\left(z_{i}\right)=\left(1+\Delta \varepsilon / \varepsilon_{i}\right)^{-1 / 4} \simeq 1-y$ is the equilibrium matter temperature and radiation temperature ratio at the beginning of the heating process (i.e. at $z_{i}$ ). Typical values of $y$ expected from reionization are $\approx 10^{-7}$. For example, for two astrophysical reionization scenarios based on different radiative feedback assumptions (filtering and suppression models) Burigana et al. (2008) found $y \simeq(0.965-1.69) \times 10^{-7}$. Other kinds of unavoidable spectral distortions are Bose-Einstein (BE)-like distorted spectra, produced by the dissipation of primordial perturbations at small scales, damped by photon diffusion and thus invisible in the CMB anisotropies, which produce a positive chemical potential, $\mu_{0} \simeq 1.4 \Delta \varepsilon / \varepsilon_{i}$, and Bose-Einstein condensation of the CMB by colder electrons associated with the faster decrease of matter temperature in the expanding Universe relative to than that of radiation, which gives a negative chemical poten-

\footnotetext{
${ }^{6} \mathrm{http}: / /$ www.core-mission.org/

${ }^{7}$ http://www.prism-mission.org/
} 
tial (for a recent review see Sunyaev \& Khatri 2013, and references therein). These two kinds of distortions are characterized by an amplitude, respectively, in the range $10^{-9}-10^{-7}$ (and in particular $\simeq 2.52 \times 10^{-8}$ for a primordial scalar perturbation spectral index $n_{S}=0.96$, without running), and $\simeq 3 \times 10^{-9}$. Since very small scales not explored by current CMB anisotropy data are relevant in this context, a wide range of primordial spectral indexes needs to be explored. A wider range of chemical potentials is found by Chluba et al. (2012), allowing also for variations of the amplitude of primordial perturbations at very small scales, as motivated by different inflation models.

The free-free signal associated with cosmological reionization represents the most relevant type of low-frequency spectral distortion. The CMB brightness temperature, $T_{b r}$, under the combined effect of Comptonization and free-free processes is approximated by $\left(T_{b r}-T_{C M B} \phi_{i}\right) / T_{C M B} \simeq$ $y_{B} / x^{2}-2 y \phi_{i}$, where $x=h v / k T_{C M B}$, and $y_{B}$ is the free-free distortion parameter. For a homogeneous ionized medium $y_{B}(x, t)=\int_{t_{i}}^{t}\left(\phi-\phi_{i}\right) \phi^{-3 / 2} g_{B}(x, \phi) K_{0 B} d t$, where $g_{B}$ is the Gaunt factor weighted over ionized atoms and the bremsstrahlung rate in a hydrogen-helium plasma is given by $K_{0 B}(z) \simeq(8 \pi / 3) e^{6} h^{2} n_{e}^{\text {free }}\left(n_{H}^{+}+n_{H e}^{+}+4 n_{H e}^{++}\right) \phi^{7 / 2} /\left[m\left(6 \pi m k T_{e}\right)^{1 / 2}\left(k T_{e}\right)^{3}\right]$ (Burigana et al. 1991). The density of free electrons and ionized atoms is determined by the reionization history. Since structure formation is far from homogeneous, the dependence of free-free emission on the square of the baryon density implies a distortion amplification with respect to the case of homogeneous medium by a large factor, $\simeq 1+\sigma^{2}$, where $\sigma^{2}$ is the matter distribution variance ( $\gg 1$ at moderate and low redshifts), as found in Trombetti \& Burigana (2014) who combine Boltzmann codes for the matter variance evaluation with a dedicated free-free distortion code. The results are shown in right panel of Fig. 1, where both free-free signal and Comptonization decrement are included.

The radio background, mainly contributed by the very bright Galactic synchrotron emission, is approximated (in terms of brightness temperature) by $(T / \mathrm{K}) \sim 2.8 \times(\mathrm{v} / \mathrm{GHz})^{-2.55}$. The extragalactic background determined by the ARCADE 2 experiment (Seiffert et al. 2011) (expressed in terms of equivalent thermodynamic temperature) is also consistent with a power law with a very similar index, -2.57 , but with an amplitude about two times smaller, plus a frequency-independent $\mathrm{CMB}$ contribution at $\simeq 2.725 \mathrm{~K}$. In order to accurately observe with dedicated experiments the tiny $\mathrm{CMB}$ spectral distortions discussed above, the problem of the modelling and subtraction of the contribution from Galactic emissions and extragalactic foreground needs to be solved. The high sensitivity and resolution of the SKA can be used to address this issue, as discussed below.

Current measurement of radio source counts at $\mathrm{GHz}$ frequencies (see e.g. Prandoni et al. 2001, Condon et al. 2012) have sensitivity levels of tens of $\mu \mathrm{Jy}$ (a recent estimation of radio source background can be found in Gervasi et al. 2008). However, the very faint tail of radio source counts and their contribution to the radio background at very low brightness temperature is not accurately known. Exploiting the recent differential number counts at $0.153 \mathrm{GHz}$ (Williams et al. 2014), 0.325 GHz (Mauch et al. 2013), 1.4 GHz (Condon et al. 2012), and $1.75 \mathrm{GHz}$ (Vernstrom et al. 2014a) it is possible to evaluate the contribution, $T_{b}$, to the radio background from extragalactic sources in various ranges of flux densities. While these signals are clearly negligible compared to the accuracy of current CMB spectrum experiments, mostly at $\lambda \gtrsim 1 \mathrm{~cm}$, they can be significant at the accuracy level potentially achievable with future experiments. Assuming to have subtracted the sources brighter than several tens of nJy, $T_{b}$ is found to be less than $\sim 1 \mathrm{mK}$ at frequencies above $\sim 1 \mathrm{GHz}$, but larger than $\sim 10 \mathrm{mK}$ below $\sim 0.3 \mathrm{GHz}$. The estimate of the minimum source detection threshold is given by the source confusion noise which, around $1.4 \mathrm{GHz}$, has been quoted by Condon et al. 

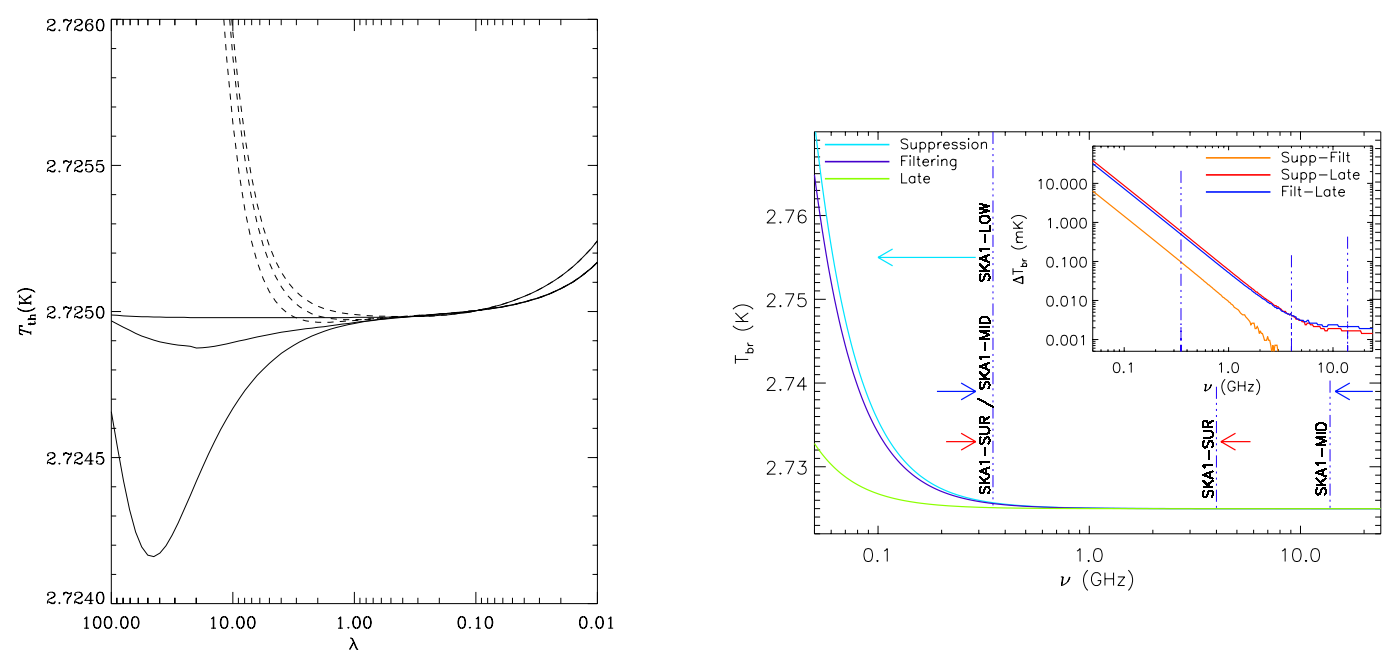

Figure 1: Left panel: $\mathrm{CMB}$ distorted spectra in terms of equivalent thermodynamic temperature as a function of the wavelength $\lambda$ (in $\mathrm{cm}$ ) in the presence of a late energy injection with $\Delta \varepsilon / \varepsilon_{i} \simeq 4 y=5 \times 10^{-6}$ plus an early/intermediate energy injection with $\Delta \varepsilon / \varepsilon_{i}=5 \times 10^{-6}$ (about 20 times smaller than current upper limits) occurring at the "time" Comptonization parameter $y_{h}=5,1,0.01$ (from the bottom to the top; in the figure the cases at $y_{h}=5$ - when the relaxation to a Bose-Einstein modified spectrum with a dimensionless chemical potential given, in the limit of small distortions, by $\mu \simeq 1.4 \Delta \varepsilon / \varepsilon_{i}$ is achieved - and at $y_{h}=1$ are extremely similar at short wavelengths; solid lines) and plus a free-free distortion with $y_{B}=10^{-6}$ (dashes). $y_{h}$ is defined by $y$ but with $T_{e}=T_{C M B}$ when the integral is computed from the time of the energy injection to the current time. From Burigana et al. (2004). Right panel: free-free distortion in the SKA2 frequency range produced by two astrophysical reionization histories (a reionization phenomenological model, late model, see Naselsky \& Chiang 2004, with parameters given by Eq. (4) of Trombetti \& Burigana 2012 is also displayed for comparison). The inset shows the absolute differences between the models. Vertical lines display the frequency ranges of the three SKA1 configurations.

(2012): $5 \sigma_{\text {conf }} \simeq 5 \times 1.2(v / 3 \mathrm{GHz})^{-0.7}\left(\theta / 8^{\prime \prime}\right)^{10 / 3} \mu \mathrm{Jy}$, where $\theta$ defines the relevant resolution. According to the authors, the finite angular extension of faint galaxies, $\theta \sim 1$ ", implies a "natural confusion limit" of about $10 \mathrm{nJy}$ at frequencies around $\sim 1.4 \mathrm{GHz}$, thus indicating that, for deep surveys such as those discussed below, source confusion will not represent a relevant limitation.

At $1 \mathrm{GHz} \lesssim v \lesssim$ some $\mathrm{GHz}(\lambda \approx 1 \mathrm{dm})$ the signal amplitudes found for CMB distorted spectra well below FIRAS constraints (see Fig. 1) are significantly larger than the estimates of the background from extragalactic sources fainter than some tens of nJy. Free-free distortion amplitude increases at decreasing frequencies, but source confusion noise may represent there a serious problem, possibly preventing the achievement of the faint detection threshold necessary to have a source contribution to the background significantly less than the CMB distortion amplitude.

Extragalactic source contribution is small compared to the Galactic radio emission, which currently represents the major astrophysical problem in CMB spectrum experiments, but, unlike the Galactic emission, it cannot be subtracted from the $\mathrm{CMB}$ monopole temperature by exploiting its angular correlation properties. Accurate absolute measurements with a wide frequency coverage can allow a joint fit of both CMB distorted spectra and astrophysical signals (see e.g. Salvaterra \& Burigana 2002 for an application to FIRAS data) but a direct radio background estimate from precise number counts will certainly improve the robustness of this kind of analyses.

The relevance of this problem emerged in the detection by the Absolute Radiometer for Cos- 
mology, Astrophysics, and Diffuse Emission $2^{8}$ (ARCADE 2) of an excess in the CMB absolute temperature at $3.3 \mathrm{GHz}$ (Singal et al. 2011, Seiffert et al. 2011), which is likely explained by a background of very faint sources or by residual emission from subtraction of the Galactic component. Singal et al. (2010) concluded that, for a radio background at the level reported, the majority of the total surface brightness would have to have been produced by ordinary star-forming galaxies at redshift $z \gtrsim 1$, characterized by an evolving radio-far-IR correlation, which changes towards the radio loud with increasing redshift. Using the JVLA at $3 \mathrm{GHz}$ Vernstrom et al. (2014b) ruled out a new discrete population peaking brighter than $50 \mathrm{nJy}$ while Vernstrom et al. (2014a) constrained the contribution to the ARCADE 2 excess from an extended population peaking above $1 \mu \mathrm{Jy}$. Analyzing dedicated radio observations with the JVLA, ${ }^{9}$ Condon et al. (2012) argued that the discrete sources possibly dominating the reported extragalactic background excess cannot be located in (or near) galaxies and must be typically fainter than $0.03 \mu \mathrm{Jy}$ at $1.4 \mathrm{GHz}$. The problem raised by this controversial $3.3 \mathrm{GHz}$ excess underlines how crucial the precise estimation of very faint source counts is for the accurate exploitation of CMB spectrum measurements.

SKA continuum surveys, driven by a set of SKA top priority science cases and defined to provide significant advances over pre-SKA surveys, are described in Prandoni \& Seymour (2015). Deep and ultra-deep surveys are clearly the most relevant ones to determine the very faint source counts, while $P(D)$ methods can be exploited to extract information on number counts below the survey sensitivity (Condon et al. 2012), particularly in low frequency continuum surveys, dedicated to non-thermal emission in clusters and filaments. The Ultra Deep survey dedicated to the Star Formation History of the Universe (SFHU), with their planned rms sensitivity of some tens of nJy per beam and arcsec or better resolution, will represent a great opportunity for an accurate determination of source number counts down to very faint fluxes, significantly helping the solution of one fundamental problem of the future generation of CMB spectrum experiments at long wavelengths.

\subsection{Free-free localized emissions}

The SKA will be able, for the first time, to trace the detailed distribution of neutral hydrogen before reionization, and the neutral-to-ionized transition state at the reionization epoch, through the 21-cm line (see e.g. Schneider et al. 2008). It is also possible to trace the development of ionized material directly by looking for the free-free emission from ionized halos. The expected signal can be calculated exploiting reionization phenomenological and astrophysical models through both semi-analytical methods (Naselsky \& Chiang 2004, Trombetti \& Burigana 2014), and numerical simulations (Ponente et al. 2011).

The direct observation of diffuse gas and Pop III objects in thermal bremsstrahlung has been investigated by Oh (1999). Observations at high resolution of dedicated sky areas are a natural way to distinguish free-free distortion by ionized halos rather than by diffuse ionized IGM. The SKA should be able to detect up to $\sim 10^{4}$ individual free-free emission sources with $z>5$ in 1 square degree (see left panel of Fig. 2).

Simulations by Ponente et al. (2011) show that the expected individual halo signal should be $S_{\mathrm{ff}} \sim 3.67 \times 10^{-9} \mathrm{Jy}$, while the analytic $\beta$-model for halo density profiles predicts a signal a factor $\sim 7.5$ larger. In terms of $\Delta T$, the maximum temperature distortion is about a few $\mu \mathrm{K}$ (at $1 \mathrm{GHz}$ )

\footnotetext{
${ }^{8}$ http://asd.gsfc.nasa.gov/archive/arcade/

${ }^{9}$ http://www.nrao.edu/pr/2012/jansky/
} 

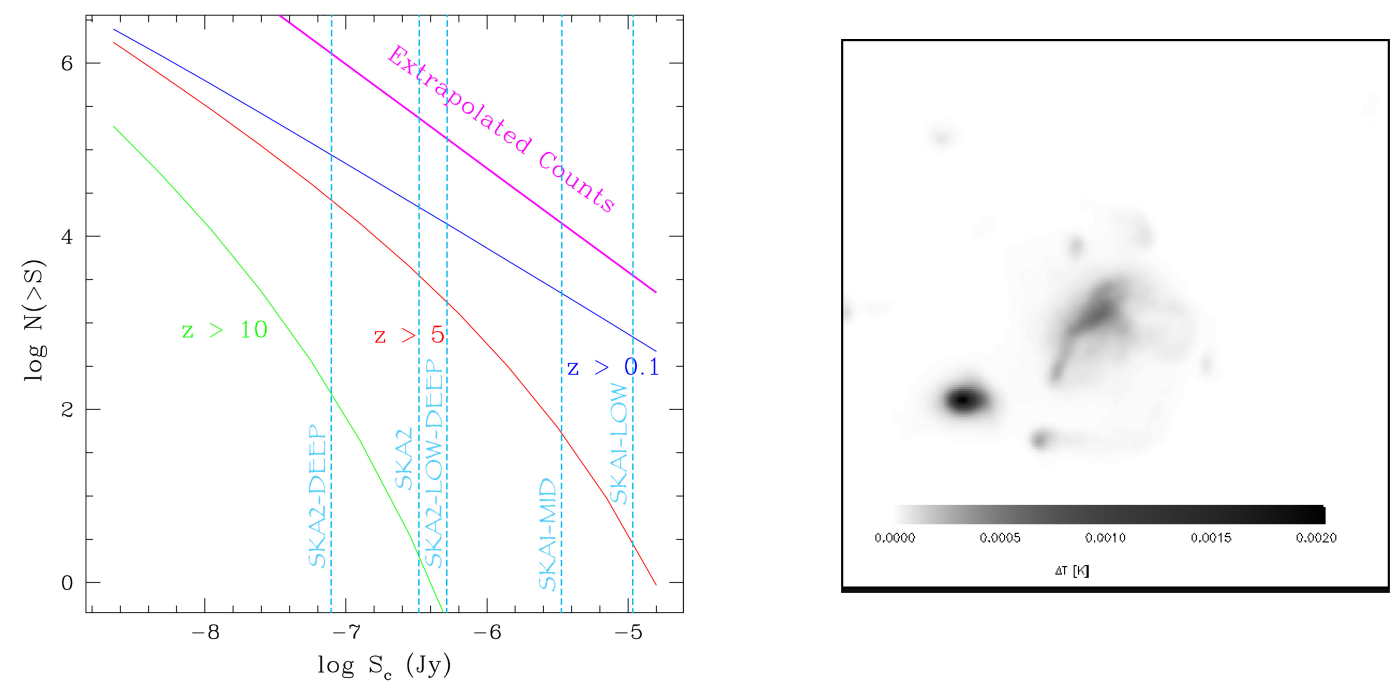

Figure 2: Left panel: number of sources which may be detected in $1 \mathrm{deg}^{2}$ by SKA, as a function of the threshold flux $S_{c}$ (theoretical predictions from Oh 1999). Realistic limiting fluxes (5 $\sigma$ sensitivity levels achievable in one hour of integration on one field of view (FoV)) for point source detection are shown (vertical lines). We report also the $(5 \sigma)$ sensitivity achievable by SKA2 with a deep exposure (in $\sim$ one day of integration) at low and mid/high frequencies. Extrapolated source counts from Partridge et al. (1997). Right panel: free-free signal for a halo with $M=6.6 \times 10^{14} h^{-1} M_{\odot}$ at $z=0.15$. The greyscale shows the signal in $\mathrm{K}$ and at $1 \mathrm{GHz}$. The field of view is $\approx 40^{\prime}$. The total flux in this region is $S_{\mathrm{ff}}=2.83 \times 10^{-5} \mathrm{Jy}$. From Ponente et al. (2011).

at the center of the cluster. More massive and denser clusters would produce stronger signals, representing a useful way to study the intracluster medium. For a simulated massive cluster at redshift $z=0.15$ the free-free distortion at $1 \mathrm{GHz}$ is of the order of $1 \mathrm{mK}$ in the cluster regions (see right panel of Fig. 2). Thus, the precise mapping of individual halos represents an interesting goal for the excellent imaging capabilities of the SKA.

\section{Cross-correlation with CMB}

High accuracy CMB surveys such as those carried out and expected by the Planck satellite ${ }^{10}$ are designed to cover high sky fractions, $f_{\text {sky }}$, or the whole sky. The SKA is mainly designed to achieve very faint fluxes on limited sky fields, but its sensitivity is so high on typical FoVs of $\sim$ degree side at frequencies $\sim 1 \mathrm{GHz}$, that it is reasonable to expect to be able to cover a significant $f_{\text {sky }}$ (thousands of square degrees) with unprecedented sensitivity in some months of integration. This will improve the cross-correlation analyses with CMB surveys and with surveys in other frequency bands (see also Takahashi et al. 2015).

\subsection{Integrated Sachs-Wolfe effect and constraints on dark energy}

The ISW effect comes from the line-of-sight integral in the Sachs Wolfe equation (Sachs \& Wolfe 1967). It arises when CMB photons streaming across the Universe interact with the timeevolving gravitational potential wells associated with foreground large-scale structure (LSS). The evolution of the potential leads to a net change of the photon energies as they pass through the LSS. The ISW is a linear effect which depends the cosmological model, since it requires a change in the

\footnotetext{
${ }^{10}$ www.rssd.esa.int/planck
} 
cosmic fluid equation of state. The evolution of the gravitational potential is related to the matter linear density perturbations; in the matter dominated regime, the growth of matter perturbation is proportional to the scale factor. This balances the dilution of matter due to the cosmic expansion and makes the gravitational potential variations negligible. They are relevant however at early times, when the Universe goes from being radiation dominated to matter dominated (early ISW), and at late times, as the dark energy (DE) (or curvature) takes over from the matter (late ISW). The ISW contribution to the CMB anisotropy in a direction $\hat{n}$ on the sky is approximately given by $\Delta^{I S W}(\hat{n}) \approx-2 \int_{\text {Last Scattering }}^{\text {Today }} d \eta \dot{\Phi}[r \hat{n}, \eta]$, where $\Phi$ is the Newtonian potential, the dot denoting a derivative with respect to the conformal time $\eta$, and $r(\eta)$ is the proper distance.

Unlike the early ISW, the late ISW is virtually uncorrelated with the CMB anisotropies generated at last scattering. Direct detection of late ISW is difficult because of its small amplitude and its dominance only on super-horizon (i.e. large) scales, where cosmic variance is large. However, it is possible to isolate the late ISW generated at low redshifts through the cross-correlation of the CMB maps with LSS surveys. Indeed, when CMB photons cross a time-varying potential, they become slightly hotter or colder: statistically, we expect a tiny correlation of hot spots in the CMB with LSS, an effect expected to be less than $1 \mu \mathrm{K}$, orders of magnitude smaller than the CMB correlations (Crittenden \& Turok 1996, Peiris \& Spergel 2000). Several measurements have been performed to detect the ISW signal: positive cross-correlations were measured using Sloan Digital Sky Survey ${ }^{11}$ (SDSS) galaxy data and WMAP (Fosalba et al. 2003, Padmanabhan et al. 2005, Granett et al. 2008, Granett et al. 2009, Papai et al. 2011), on APM galaxies (Fosalba \& Gaztanaga 2004), the 2MASS survey (Afshordi et al. 2004), and on radio data (see Nolta et al. 2004, Raccanelli et al. 2008 and Boughn \& Crittenden 2004a,b where correlations with hard X-ray background were found). Also, Afshordi et al. (2004), Rassat et al. (2007), and Francis \& Peacock (2010) used IR galaxy samples to characterize the ISW signal. The typical significance of ISW detections is currently quite low, around 2-3 $\sigma$. The cross-correlation detection of CMB with LSS requires a good CMB map on large scales and a deep enough galaxy distribution map with large $f_{\text {sky }}$ to reduce the uncorrelated CMB map noise, with $S / N \propto f_{\text {sky }}^{1 / 2}$.

Given a CMB map in temperature and a galaxy survey $\mathbf{x}=(\mathbf{T}, \mathbf{G})$ (vector in pixel space), the quadratic maximum likelihood (QML) estimator (Tegmark 1997) provides an estimate of the angular power spectrum (APS) $\hat{C}_{\ell}^{X}$, where $X=T T, T G, G G$. QML is well suited for such analysis, being optimal, i.e. unbiased and with minimum variance, an essential feature when the $\mathrm{S} / \mathrm{N}$ ratio is low, as for the ISW effect. It is computationally demanding, but can be applied at relatively low resolution, i.e. for large scales, where the (late) ISW effect appears, and even for $f_{s k y}<1$, being a pixel based method. A set of estimators (Xia et al. 2009) exploiting the features of the redshift surveys considered has been developed: the correlation of WMAP7 CMB data with radio sources in the NRAO Very Large Array Sky Survey ${ }^{12}$ (NVSS) and its implications on the cosmological perturbation statistics (Xia et al. 2010b); the foreground removal from CMB maps (Xia et al. 2010a) to improve auto and cross-correlation spectra (Xia et al. 2011); and WMAP7 maps and NVSS cross-correlations with dedicated methods to constrain the DE content (Schiavon et al. 2012).

From the 2013 data release, the Planck Collaboration detected the ISW effect with a $2 \sigma-$

\footnotetext{
${ }^{11}$ www.sdss.org

${ }^{12} \mathrm{http} / / /$ www.cv.nrao.edu/nvss/
} 
$4 \sigma$ significance, depending on the adopted method (Planck Collaboration 2014a). The SKA will play a crucial role in improving the current large-scale galaxy dataset. The requirement to have a negligible shot noise in the galaxy power spectrum is $\beta$ FoV $\mathrm{T}_{0}>10 \mathrm{deg}^{2} \mathrm{yr}$ (Abdalla \& Rawling 2005); here $\beta$ is the receiver bandwidth, $T_{0}$ is the survey duration and FoV is $1 \mathrm{deg}^{2}$ and $200 \mathrm{deg}^{2}$ for SKA1-MID and SKA2-LOW, respectively. It is foreseen that a 1-yr SKA survey will contain $>10^{9}\left(f_{\text {sky }} / 0.5\right) \mathrm{HI}$ galaxies in a redshift range $0<z<1.5$. This makes the combination of SKA and Planck data a powerful tool for investigating the ISW correlation, offering the opportunity to achieve an independent measurement of the effect and increasing the confidence level in the detection from the present, marginal evidence. It will also be possible to improve constraints on the statistics of primordial perturbations and DE dynamics.

\subsection{Non-Gaussianity from joint analyses of CMB and radiosources}

Statistical analyses of the extragalactic source distribution can probe the Gaussianity of primordial perturbations through their imprints at the origin of LSS. Different kinds of non-Gaussianity, such as the local type (loc), equilateral (eq), enfolded (enf), orthogonal, have been predicted (Bartolo et al. 2004, Komatsu et al. 2010). For example, the local type is parameterized by a constant dimensionless parameter $f_{N L}$ (see e.g. Verde et al. 2000, Komatsu \& Spergel 2001, Babich et al. 2004), $\Phi=\phi+f_{N L}\left(\phi^{2}-<\phi^{2}>\right.$ ), where $\Phi$ denotes Bardeen's gauge-invariant potential (evaluated deep in the matter era in the CMB convention) and $\phi$ is a Gaussian random field. Extragalactic radio sources are powerful tracers of the LSS, since they span a large volume extending out to substantial redshift. A global analysis of the constraints on the amplitude of primordial non-Gaussianity (PNG) from the APS obtained from extragalactic radio sources (the SDSS Release Six quasar catalogue), the final SDSS II Luminous Red Galaxy (LRG) photometric redshift survey, and cross-correlation with the WMAP CMB temperature map, has set limits of $f_{N L}^{l o c}=48 \pm 20, f_{N L}^{e q}=50 \pm 265$ and $f_{N L}^{e n f}=183 \pm 95$ at $68 \%$ confidence level, almost stable with respect to potential systematic errors and analysis details (Xia et al. 2011). A recent, interesting analysis of PNG of local type using the clustering of $8 \times 10^{5}$ photometric quasars from the SDSS in the redshift range $0.5<z<3.5$ has been presented by Leisted et al. (2014). The authors separate the sample of quasars into four redshift bins by selecting objects with photometric redshift estimates and mitigate the impact of systematics in the estimate of power spectra with a novel technique, the extended mode projection, based on the measurement and mapping onto the sky of the most potential systematics (e.g. observing conditions, calibration) during SDSS observations. They obtain $-49<f_{N L}^{l o c}<31$ and predict an error $\sigma\left(f_{N L}^{l o c}\right) \simeq 5$ from the angular power spectra of galaxies in 20 tomographic bins in the redshift range $0.5<\mathrm{z}<3.5$ that will be obtained with the Large Synoptic Survey Telescope-like photometric survey (LSST DE Science Collaboration 2012). Furthermore, a robust constraint, $f_{N L}^{l o c}=5 \pm 21$, on PNG of local type has been derived by Giannantonio et al. (2014) cross-correlating a wide set of currently available catalogs of galaxy surveys and them with WMAP maps and performing an extended analysis of the possible systematics aimed at reducing their impact on the results. Tests of non-Gaussianity would have profound implications for inflationary mechanisms - such as single-field slow roll, multifields, curvaton (local type) - and for models whose effects on the halo clustering can be described by the equilateral template (related to higher-order derivative type non-Gaussianity) and by the enfolded template (related to modified initial state or higher-derivative interactions). Planck data already set strong limits on the non-Gaussianity parameter $f_{N L}$, namely 

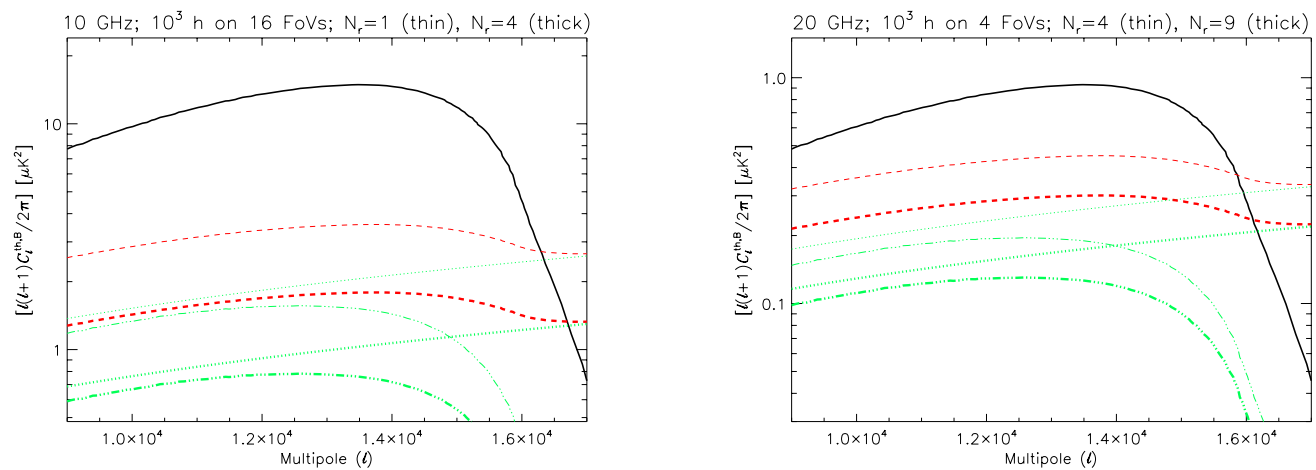

Figure 3: B-mode APS of the CMB at $10 \mathrm{GHz}$ and $20 \mathrm{GHz}$ induced by the Faraday rotation field with PMF normalization comoving scale $\lambda=1 \mathrm{Mpc}$ and $n_{B}=0$ (solid black line - adapted from Fig. 2 in Kosowsky et al. 2005) compared with SKA2 sensitivity (red dashes) achieved in $\sim 10^{3}$ hours of integration on a suitable number of FoVs, each of area $\simeq 0.49 \times(1.67 \mathrm{GHz} / v)^{2} \mathrm{deg}^{2}$. Cosmic+sampling variance from this signal (green three-dots) and instrumental noise limitation (green dots) are also separately displayed. A $10 \%$ binning in $\ell$ is assumed. With relatively short baselines exploited here, the sharing of the same integration time on a number of FoVs may be more advantageous in terms of trade-off between the minimization of sampling and noise variances. The use of a focal-plane array with a number of receivers, $N_{r}$, allowing to observe a correspondingly larger sky area in the same time, will imply a better signal-to-noise ratio. See also the text.

$f_{N L}^{l o c}=2.7 \pm 5.8, f_{N L}^{e q}=-42 \pm 75$ and $f_{N L}^{e n f}=-25 \pm 39$ at $68 \%$ confidence level (Planck Collaboration 2014b), significantly constraining or ruling out many classes of inflationary models.

Recent forecast works exploit the SKA radio continuum surveys (Prandoni \& Seymour 2015) covering $\simeq 3.1 \times 10^{4} \mathrm{deg}^{2}$ out to high redshift. To mitigate the problem of lack of redshift data, Raccanelli et al. (2014) propose a combination of two methods: cross-correlation of the radio counts with CMB temperature anisotropies, i.e. the ISW effect, to reduce systematics on large scales which are particularly sensitive to PNG; and cross-identification of radio sources with optical data in order to split the radio sources into redshift bins. The authors show that even with only two redshift bins, a tomographic analysis could improve the constraints on $f_{N L}^{l o c}$ by an order of magnitude with respect to the case of a single redshift bin, achieving $\sigma\left(f_{N L}^{l o c}\right) \simeq 2$, while including full redshift information will allow highly precise measurements of the non-Gaussianity parameter, with $\sigma\left(f_{N L}^{l o c}\right)<1$. General relativistic effects on the galaxy number counts, such as a non-linear primordial correction and linear projection effects from observing in redshift space on the past light-cone, have been included in the analysis of Camera et al. (2014). Their relevance emerges for a precise analysis of small values of $f_{N L}^{l o c}$, necessary in the light of Planck results. Neglecting difficulties due to cosmological parameter degeneracies, since the inclusion of PNGs does not significantly broaden the constraints on other parameters, the standard approach is adopted of varying freely only the parameters one is interested in and fixing all the others to their fiducial values, as fitted for instance by Planck. The authors find that with SKA2 (in its full configuration) it will possible to constrain $f_{N L}^{l o c}$ down to $\sigma\left(f_{N L}^{l o c}\right) \simeq 1.54$, thanks to the large number of HI galaxies that will be detected up to high redshift. These works indicate the possibility to improve with SKA the constraints on $f_{N L}^{l o c}$ of a factor $\sim 3$ with respect to Planck results.

The combination of high accuracy and deep extragalactic source surveys achievable with the SKA and the forthcoming and future CMB maps will provide a significant progress in this topic, at least for the local configuration. 


\section{Primordial magnetic fields versus seed magnetic field}

The large-scale magnetic fields of the order of few $\mu \mathrm{G}$ observed in galaxies and galaxy clusters may be the product of the amplification, during structure formation, of primordial magnetic seeds (Ryu et al. 2012). Several early-universe models predict the generation of primordial magnetic fields (PMF), either during inflation or during cosmological phase transitions (see Widrow et al. 2012 for a review). The impact of PMF on Big Bang nucleosynthesis constrains their amplitude at the $\mu \mathrm{G}$ level (Kawasaki \& Kusakabe 2012). Tighter constraints at the $\mathrm{nG}$ level come from CMB temperature APS (Paoletti \& Finelli 2011, Shaw \& Lewis 2012, Paoletti \& Finelli 2013) and bispectrum (Caprini et al. 2009, Seshadri \& Subramanian 2009, Trivedi et al. 2010, Trivedi et al. 2012, Shiraishi et al. 2012). PMF also impact structure formation. The presence of an extra component of anisotropic stress carried by the PMF and the Lorentz force induced on baryons modifies the evolution of matter perturbations, and impacts the small-scale matter power spectrum, and the formation and early evolution of structure. This effect, studied through magnetohydrodynamic $N$-body numerical simulations (Dolag 2006), is still at an early stage of development, especially concerning the analytical non-linear treatments. Data from the $\gamma$-ray observatory Fermi have recently added new intriguing observations in the context of cosmological magnetic fields which might be interpreted as a lower bound on the PMF amplitude. The data on $\gamma$-ray cascades from blazars show a lack of photons, which is compatible with diffuse extra-galactic magnetic fields in the intracluster medium (voids) with a lower bounds of the order of $10^{-15}-10^{-16} \mathrm{G}$ (Neronov \& Vovk 2010, Taylor et al. 2011, Vovk et al. 2012). If this lower bound on the PMF is be confirmed, the SKA could perform crucial measurements towards probing the PMF generation mechanism. Current CMB constraints on the PMF are dominated by temperature anisotropy accuracy measurements, since PMF impact the high multipoles $(\ell \sim 1500)$ without suppression by Silk damping. Recent measurements from WMAP combined with SPT and Planck were crucial in disentangling PMF contributions from high- $\ell$ foreground and secondary anisotropies (Paoletti \& Finelli 2013). SKA measurements in temperature and polarization of very high- $\ell$ multipoles could improve these bounds on the PMF as well as the characterization of foreground and secondary anisotropies beyond the Silk damping tail. The PMF contribution to CMB anisotropies is generated either at the last-scattering surface or by Faraday rotation of the intervening magnetic fields of the stochastic background with the characteristic frequency dependence $\propto v^{-4}$ (Kosowsky \& Loeb 1996). The smoking gun of the Faraday rotation from a stochastic background of PMF is a $B$-polarization signal at very high- $\ell$ multipoles, with a peak around $\ell \sim 1.4 \times 10^{4}$ (Kosowsky et al. 2005). SKA2 (in particular with the bands at $\sim 10-20 \mathrm{GHz}$, because of their minor foreground contamination) can target such signal (see Fig. 3 ) in the multipole range $\ell \sim 10^{4}-1.5 \times 10^{4}$ for a magnetic field amplitude $\approx \mathrm{nG}$ allowed by the temperature measurement. We exploit the flexibility of SKA to identify suitable conditions for detecting this signal, searching for configurations able to jointly minimize sampling and noise variances. We find that, even assuming a collecting area (and, correspondingly, receiver numbers) decreased to about $50 \%$, relatively short baselines (around $5 \mathrm{~km}$ ) are better suited to this aim. While the FoV at $\sim 10 \mathrm{GHz}$ is in principle large enough for a detection (or to improve current constraints on PMF models), the implementation of SKA2 with focal-plane arrays, allowing an increase the observed sky area in the same integration time, will be extremely useful for this research, particularly at $20 \mathrm{GHz}$. Obviously, the best configuration will be selected according to the 
allocated time and actual implementation of SKA2. CMB polarization can be crucial to determine or constrain the nature of the stochastic background, given the different dependence of the Faraday effect on $B_{\lambda}$ and the magnetic field power spectrum index $n_{B}$.

\section{Galactic foregrounds}

For many of the above topics the accurate study of Galactic emission is particularly crucial (see e.g. Burigana et al. 2013). The possibility of mapping intermediate and large scales with the SKA relies on the ability to merge different FoVs into maps with appropriate large-scale calibration and matching, possibly in combination with other radio surveys. On the other hand, the relatively bright Galactic radio signal does not require the extreme sensitivity demanded, for instance, by CMB fluctuation mapping at the SKA highest frequencies. SKA1 and SKA2 observations in the radio domain will allow us to test Galactic synchrotron emission models, 3D physical models of the Galaxy and the large scale coherent component of the Galactic magnetic field (Sun et al. 2008, Sun \& Reich 2009, Sun \& Reich 2010, Fauvet et al. 2011, Fauvet et al. 2012), based on advanced numerical codes (Strong \& Moskalenko 1998, Waelkens et al. 2009) and including turbulence phenomena (Cho \& Lazarian 2002).

For both Galactic science and the treatment of foregrounds in cosmology, it is also important to improve our understanding of the anomalous microwave emission (AME) and of the haze component. AME is the recently identified emission component which is well-correlated with far-IR dust emission. It is produced by rapidly spinning small dust grains having an electric dipole moment (Draine \& Lazarian 1998) and its spectrum is expected to peak in the range 15-50 GHz. For the first time Planck was able to define the shape of the spectrum on the high frequency side of the emission peak in a number of dust/molecular/HII regions (Planck Collaboration 2011). In the frequency range $20-40 \mathrm{GHz}$ AME is typically comparable in brightness to the free-free for the inner Galactic plane. SKA2 could provide precise mapping on the low frequency tail of this emission.

Planck was also able to identify and characterize the emission from the Galactic haze at microwave wavelengths (Planck Collaboration 2013). This is a distinct component of diffuse Galactic emission, roughly centered on the Galactic centre, extended to $|b| \sim 35^{\circ}$ in Galactic latitude and $|l| \sim 15^{\circ}$ in longitude. By combining WMAP and Planck data, Planck Collaboration (2013) were able to determine the spectrum of this emission to high accuracy, unhindered by the large systematic biases present in previous analyses. The derived spectrum is consistent with power-law emission with a spectral index of $-2.55 \pm 0.05$, thus excluding free-free emission as the source and instead favoring hard-spectrum synchrotron radiation from an electron population with a distribution (number density per energy) $d N / d E \sim E^{-2.1}$. At Galactic latitudes $|b|<30^{\circ}$, the microwave haze morphology is consistent with that of the Fermi $\gamma$-ray haze or bubbles (see also Carretti et al. 2012), indicating that we have a multi-wavelength view of a distinct component of our Galaxy. Given the very hard spectrum and the extended nature of the emission, it is unlikely that the haze electrons result from supernova shocks in the Galactic disk. Instead, a new mechanism for cosmicray acceleration in the centre of our Galaxy is implied. With the SKA multifrequency mapping in total intensity and polarization we will have the opportunity to firmly constrain these models.

In general, a large sky coverage is crucial for mapping Galactic radio emissions. Among the SKA continuum surveys (Prandoni \& Seymour 2015), we compare the sensitivity (on the same 
resolution element) of the $\simeq 75 \%$ sky coverage surveys at $1.4 \mathrm{GHz}$ and at $0.12 \mathrm{GHz}$, planned for 1-2 years of integration and dedicated respectively to strong gravitational lensing and legacy/rare serendipity and to non-thermal emission in clusters and filaments, with that of radio surveys (La Porta et al. 2008) currently adopted as ancillary maps in CMB experiment analyses. The former will have a sensitivity about 20 times better than the available all-sky radio survey at $1.4 \mathrm{GHz}$, the latter will have sensitivity about 4 times better than the Haslam map at $408 \mathrm{MHz}$ (Haslam et al. 1982), thus representing a significant improvement with respect to current ancillary radio maps.

Summarizing, the SKA high resolution maps of the Galactic emission will contribute to a better understanding of the Galactic foreground and will provide key astrophysical information for the separation of CMB and the cosmological $\mathrm{HI} 21 \mathrm{~cm}$ emission.

Acknowledgements - It is a pleasure to thank Isabella Prandoni for useful discussions about SKA performance and flexibility. We warmly thank the reviewer and the SKA editors for constructive comments. Partial support by ASI/INAF Agreement 2014-024-R.0 for the Planck LFI Activity of Phase E2 is acknowledged.

\section{References}

Abdalla, F.B, Rawling, S., 2005, MNRAS, 360, 27

Afshordi, N., Loh, Y.S., Strauss, M.A., 2004, Phys. Rev. D, 69, 083524

Babich, D., Creminelli, P., Zaldarriaga, M., 2004, JCAP, 08, article id. 009

Bartlett, J.G., Silk, J., 1990, ApJ, 353, 399

Bartlett, J.G., Stebbins, A., 1991, ApJ, 371, 8

Bartolo, N., Komatsu, E., Matarrese, S., Riotto, A., 2010, Physics Report, 402, 103

Boughn, S., Crittenden, R., 2004a, Nature, 427, 45

Boughn, S.P., Crittenden, R.G., 2004b, ApJ, 612, L64

Braun, R., 2014, "SKA1 Imaging Science Performance“, SKA-TEL-SKO-DD-XXX, Rev. A Draft 2

Burigana, C., Danese, L., de Zotti, G., 1991, A\&A, 246, 59

Burigana, C., Davies, R.D., de Bernardis, P., et al., 2013, Int. J. Modern Phys. D, 22, 1330011

Burigana, C., de Zotti, G., Danese, L., 1995, A\&A, 303, 323

Burigana, C., de Zotti, G., Feretti, L., 2004, New Astronomy Reviews, 48, 1107

Burigana, C., Popa, L.A., Salvaterra, R., et al., 2008, MNRAS, 385, 404

Burigana, C., Salvaterra, R., 2003, MNRAS, 342, 543

Camera, S., Santos, M.G., Maartens, R., 2014, arXiv:1409.8286v1

Caprini, C., Finelli, F., Paoletti, D., Riotto, A., 2009, JCAP, 06, article id. 21

Carretti, E., Crocker, R.M., Staveley-Smith, L., et al., 2012, Nature, 493, 66

Carr, B., Kohri,K., Sendouda, Y., Yokoyama, J., 2010, Phys. Rev. D, 81, 104019

Chluba, J., Erickcek, A.L., Ben-Dayan, I., 2012, ApJ, 758, 76

Chluba, J., Sunyaev, R.A., 2012, MNRAS, 419, 1294

Cho, J., Lazarian, A., 2002, ApJ, 575, L63

Condon, J.J., Cotton, W.D., Fomalont, E.B., et al., 2012, ApJ, 758, article id. 23

COrE Collaboration, 2011, "COrE - Cosmic Origins Explorer - A White Paper", arXiv:1102.2181

Crittenden, R.G., Turok, N., 1996, PRL, 76, 575 
Danese, L., Burigana, C., 1994, Lect. Notes Phys., 429, 28

Danese, L., de Zotti, G., 1980, A\&A, 84, 364

Dewdney, P., Turner, W., Millenaar, R., et al., 2013, "SKA1 System Baseline Design“, SKA-TELSKO-DD-001, Rev. 1

Dolag, K., 2006, Astronomische Nachrichten, 327, 575

Draine, B.T., Lazarian, A., 1998, ApJ, 494, L19

Ejlli, D., Dolgov, A.D., 2014, Phys. Rev. D, 90, 063514

Fauvet, L., Macias-Perez, J.F., Aumont, J., et al., 2011, A\&A, 526, A145

Fauvet, L., Macias-Perez, J.F., Désert, F.X., 2012, Astroparticle Physics, 36, 57

Fixsen, D.J., Cheng, E.S., Gales, J.M., et al., 1996, ApJ, 473, 576

Fixsen, D.J., Mather, J.C., 2002, ApJ, 581, 817

Fosalba, P., Gaztaœnaga, E., Castander, F.J., 2003, ApJ, 597, L89

Fosalba, P., Gaztanaga, E., 2004, MNRAS, 350, L37

Francis, C.L., Peacock, J.A., 2010, MNRAS, 406, 2

Gervasi, M., Tartari, A., Zannoni, M., Boella, G., Sironi, G., 2008, ApJ, 682, 223

Giannantonio, T., Ross, A.J., Percival, W.J., 2014, Phys. Rev. D, 89, 023511

Granett, B.R., Neyrinck, M.C., Szapudi, I., 2008, ApJ, 683, L99

Granett, B.R., Neyrinck, M.C., Szapudi, I., 2009, ApJ, 701, 414

Haslam, C.G.T., Salter, C.J., Stoffel, H., Wilson, W.E. 1982, A\&AS, 47, 1

Jedamzik, K.,Katalinic V., Olinto, A.V., 2000, PRL, 85, 700

Kawasaki, M., Kusakabe, M., 2012, Phys. Rev. D, 86, 063003

Khatri, R., Sunyaev, R.A., 2012, JCAP, 09, article id. 016

Kogut, A., 1996, in XVI Moriond Astrophysics meeting, March 16-23, Les Arcs, France, astro$\mathrm{ph} / 9607100$

Kogut, A., 2003, New Astronomy Reviews, 47, 945

Kogut, A., Fixsen, D.J., Chuss, D.T., et al., 2011, JCAP, 07, article id. 025

Komatsu, E., Afshordi, N., Bartolo, N., et al., 2010, in Astro2010: The Astronomy and Astrophysics Decadal Survey, Science White Papers, Vol. 158, arXiv:0902.4759

Komatsu, E., Spergel, D.N., 2001, Phys. Rev. D, 63, 063002

Kosowsky, A., Kahniashvili, T., Lavrelashvili, G., Ratra, B., 2005, Phys. Rev. D, 71, 043006

Kosowsky, A., Loeb, A., 1996, ApJ, 469, 1

Kurz, R., Shaver, P., 1999, Messenger, 96, 7

La Porta, L., Burigana, C., Reich, W., Reich, P., 2008, A\&A, 479, 641

Leistedt, B, Hiranya, H.V., Roth, N., 2014, arXiv:1405.4315v3

LSST Dark Energy Science Collaboration, 2012, arXiv:1211.0310

Mather, J.C., Cheng, E.S., Eplee, R.E. Jr., et al., 1990, ApJ, 354, L37

Mauch, T., Klöckner, H.R., Rawlings, S., et al., 2013, MNRAS, 435, 650

Naselsky, P., Chiang, L.Y., 2004, MNRAS, 347, 921

Neronov, A., Vovk, I., 2010, Science, 328, 73

Nolta, M.R.,Wright, E.L., Page, L., et al., 2004, ApJ, 608, L10

Oh, S.P., 1999, ApJ, 527, 16

Ostriker, J.P., Thompson, C., ApJ, 323, L97

Padmanabhan, N., Hirata, C.M., Seljak, U., et al., 2005, Phys. Rev. D, 72, 043525 
Pani, P., Loeb, A., 2013, Phys. Rev. D, 88, 041301

Paoletti, D., Finelli, F., 2011, Phys. Rev. D, 83, 123533

Paoletti, D., Finelli, F., 2013, Phys. Lett. B, 726, 45

Papai, P., Szapudi, I., Granett, B.R., 2011, ApJ, 732, L27

Partridge, R.B., Richards, E.A., Fomalont, E.B., Kellerman, K.I., Windhorst, R., 1997, ApJ, 483, 38

Peiris, H.V., Spergel, D.N., 2000, ApJ, 540, 605

Planck Collaboration, 2011, A\&A, 536, A20

Planck Collaboration, 2013, A\&A, 554, A139

Planck Collaboration, 2014a, Planck 2013 results. XIX, A\&A, 571, A18

Planck Collaboration, 2014b, Planck 2013 results. XXIV, A\&A, 571, A24

Ponente, P.P., Diego, J.M., Sheth, R.K., et al., 2011, MNRAS, 410, 2353

Prandoni, I., Gregorini, L., Parma, P., et al., 2001, A\&A, 365, 392

Prandoni, I. \& Seymour, N., 2015, "Revealing the Physics and Evolution of Galaxies and Galaxy Clusters with SKA Continuum Surveys", in proc. Advancing Astrophysics with the Square Kilometre Array, PoS(AASKA14)067

PRISM Collaboration, 2014, JCAP, 02, article id. 006

Procopio, P., Burigana, C., 2009, A\&A, 507, 1243

Raccanelli, A., Bonaldi, A., Negrello, M., et al., 2008, MNRAS, 386, 2161

Raccanelli, A., Doré, O., David J. Bacon, D.J., et al., 2014, arXiv:1406.0010v1

Rassat, A., Land, K., Lahav, O., Abdalla, F.B., 2007, MNRAS, 377, 1085

Ryu, D., Schleicher, D.R.G., Treumann, R.A., Tsagas, C.G., Widrow, L.M., 2012, Space Sci. Rev., 166,1

Sachs, R.K., Wolfe, A.M., 1967, ApJ, 147, 73

Salvaterra, R., Burigana, C., 2002, MNRAS, 336, 592

Schiavon, F., Finelli, F., Gruppuso, A., et al., 2012, MNRAS, 427, 3044

Schneider, R., Salvaterra, R., Choudhury, T.R., et al., 2008, MNRAS, 384, 1525

Seiffert, M.D., Fixsen, D.J., Kogut, A., et al., 2011, ApJ, 734, article id. 6

Seshadri, T.R., Subramanian, K., 2009, PRL, 103, 081303

Shaw, J.R., Lewis, A., 2012, Phys. Rev. D, 86, 043510

Shiraishi, M., Nitta, D., Yokoyama, S., Ichiki, K., 2012, JCAP, 03, article id. 41

Singal, J., Fixsen, D., Kogut, A., et al., 2011, ApJ, 730, article id. 138

Singal, J., Stawarz, L., Lawrence, A., Petrosian, V., 2010, MNRAS, 409, 1172

Stebbins, A., Silk, J., 1986, ApJ, 169, 1

Strong, A.W., Moskalenko, I.V., 1998, ApJ, 509, 212

Sun, X.H., Reich, W., Waelkens, A., Ensslin, T.A., 2008, A\&A, 477, 573

Sun, X.H., Reich, W., 2009, A\&A, 507, 1087

Sun, X.H., Reich, W., 2010, Research in Astron. Astrophys., 10, 1287

Sunyaev, R.A., Khatri, R., 2013, Int. J. Modern Phys. D, 22, 1330014

Sunyaev, R.A., Zeldovich, Ya.B., 1970, Ap\&SS, 7, 20

Takahashi, K., Brown, M.L., Burigana, C., et al., 2015, "Overview of Complementarity and Synergy with Other Wavelengths in Cosmology in the SKA era", in proc. Advancing Astrophysics with the Square Kilometre Array, PoS(AASKA14)0159 
Taylor, A.M., Vovk, I., Neronov, A., 2011, A\&A, 529, A144

Tegmark, M., 1997, Phys. Rev. D, 55, 5895

Trivedi, P., Seshadri, T.R., Subramanian, K., 2012, PRL, 108, 231301

Trivedi, P., Subramanian, K., Seshadri, T.R., 2010, Phys. Rev. D, 82, 123006

Trombetti, T., Burigana, C., 2012, J. Modern Phys., 3, 1918

Trombetti, T., Burigana, C., 2014, MNRAS, 437, 2507

Verde, L., Wang, L.M., Heavens, A., Kamionkowski, M., 2000, MNRAS, 313, L141

Vernstrom, T., Norris, Ray P., Scott, D., Wall, J.V., 2014a, arXiv:1408.4160v1

Vernstrom, T., Scott, D., Wall, J.V., et al., 2014b, MNRAS, 404, 2791

Vovk, I., Taylor, A.M., Semikoz, D., Neronov, A., 2012, ApJ, 747, L14

Waelkens, A., Jaffe, T., Reinecke, M., Kitaura, F.S., Ensslin, T.A., et al., 2009, A\&A, 495, 697

Widrow, L.M., Ryu, D., Schleicher, D.R.G., et al., 2012, Space Sci. Rev., 166, 37

Williams, W.L., Intema, H.T., Röttgering, H.J.A., 2013, A\&A, 549, A55

Xia, J.Q., Baccigalupi, C., Matarrese, S., Verde, L., Viel, M., 2011, JCAP, 08, article id. 033

Xia, J.Q., Bonaldi, A., Baccigalupi, C., 2010a, JCAP, 08, article id. 013

Xia, J.Q., Viel, M., Baccigalupi, C., Matarrese, S., 2009, JCAP, 09, article id. 003

Xia, J.Q., Viel, M., Baccigalupi, C., et al., 2010b, ApJ, 717, L17 